\title{
INHALTSVERZEICHNIS
}

\author{
TEIL 1 \\ Elementare Theorie
}

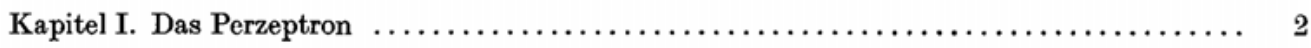

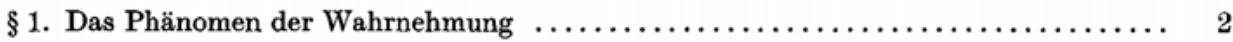

$\S 2$. Das physiologische Modell der Wahrnehmung $\ldots \ldots \ldots \ldots \ldots \ldots \ldots \ldots \ldots \ldots \ldots, 3$

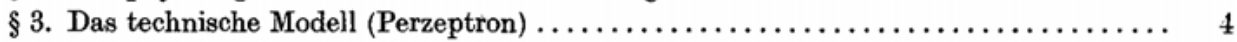

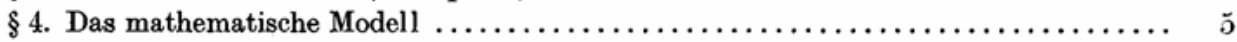

$\S 5$. Verallgemeinertes mathematisches Modell $\ldots \ldots \ldots \ldots \ldots \ldots \ldots \ldots \ldots \ldots \ldots, 7$

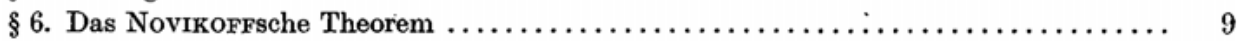

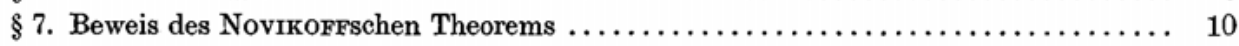

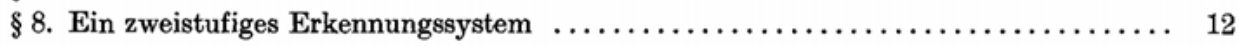

Kapitel II. Das Problem der Belehrung von Erkennungssystemen $\ldots \ldots \ldots \ldots \ldots \ldots \ldots \ldots \ldots$

$\S 1$. Das Simulationsproblem $\ldots \ldots \ldots \ldots \ldots \ldots \ldots \ldots \ldots \ldots \ldots \ldots \ldots \ldots \ldots \ldots \ldots \ldots \ldots \ldots, 15$

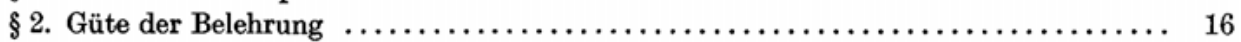

§ 3. Allgemeingültigkeit des Lernergebnisses $\ldots \ldots \ldots \ldots \ldots \ldots \ldots \ldots \ldots \ldots \ldots \ldots, 17$

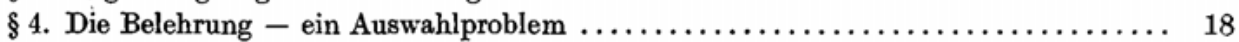

$\S 5$. Zwei Teilprobleme der Konstruktion lernfähiger Systeme $\ldots \ldots \ldots \ldots \ldots \ldots \ldots \ldots, 18$

$\S 6$. Mathematische Formulierung des Belehrungsproblems $\ldots \ldots \ldots \ldots \ldots \ldots \ldots \ldots \ldots, 19$

$\S 7$. Wege zur Lösung des Problems der Minimierung mittlerer Kosten $\ldots \ldots \ldots \ldots \ldots \ldots$ 21

§ 8. Methoden zur Minimierung der mittleren Kosten in der lernabhängigen Erkennung . 24

Kapitel III. Belehrung durch Schätzung der Wahrscheinlichkeitsverteilungen $\ldots \ldots \ldots \ldots \ldots \ldots$.

§1. Allgemeines zur Schätzung der Wahrscheinlichkeitsverteilung $\ldots \ldots \ldots \ldots \ldots \ldots \ldots, 25$

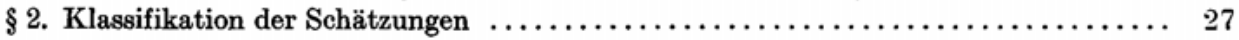

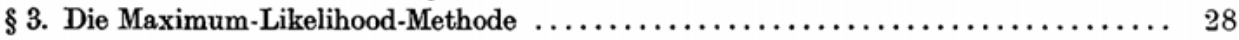

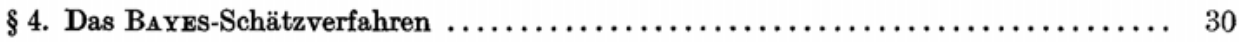

§ 5. Gegenüberstellung der BAXES- und der Maximum-Likelihood-Schätzmethode $\ldots \ldots \quad 32$

§6. Schätzung der Parameter der Verteilungsfunktionen für diskrete, unabhängige Merk-

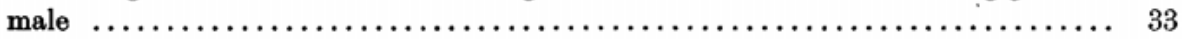

§ 7. Die BAYES-Schätzung der Verteilungsparameter diskreter, unabhängiger Merkmale . 35

§ 8. Schätzung der Parameter der Normalverteilung nach der Maximum-Likelihood-

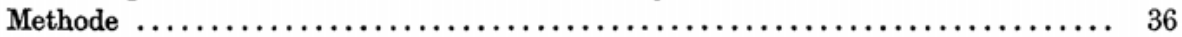

§ 9. Die BAYES-Methode zur Schätzung von Normalverteilungen $\ldots \ldots \ldots \ldots \ldots \ldots \ldots, 39$ 
Kapitel IV. Iterative Belehrungsalgorithmen für Erkennungssysteme $\ldots \ldots \ldots \ldots \ldots \ldots \ldots \ldots, 42$

$\S 1$. Die Methode der stochastischen Approximation $\ldots \ldots \ldots \ldots \ldots \ldots \ldots \ldots \ldots \ldots, 42$

$\S 2$. Deterministische und stochastische Problemstellung der Belehrung von Erkennungs-

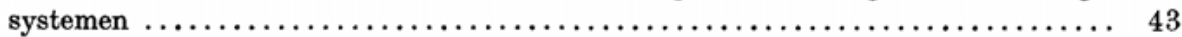

§ 3. Die in endlicher Schrittzahl konvergierenden Iterationsverfahren $\ldots \ldots \ldots \ldots \ldots \ldots, 46$

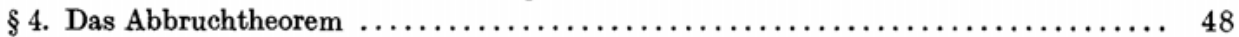

$\S 5$. Methode des zyklischen Angebots einer Lernfolge $\ldots \ldots \ldots \ldots \ldots \ldots \ldots \ldots \ldots, \quad 50$

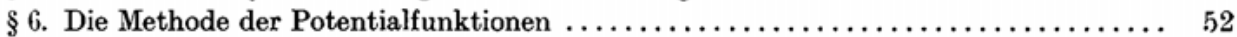

Kapitel V. Algorithmen zur Minimierung der empirischen Kosten $\ldots \ldots \ldots \ldots \ldots \ldots \ldots \ldots . \quad 54$

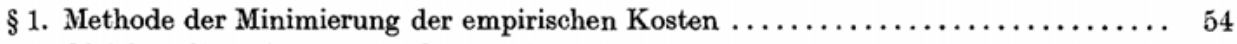

$\S 2$. Gleichmäßige Konvergenz der Häufigkeiten von Ereignissen gegen ihre Wahrschein-

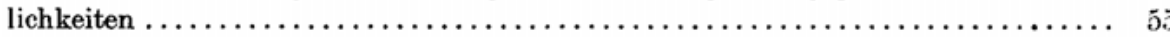

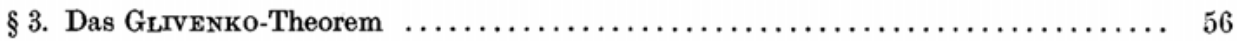

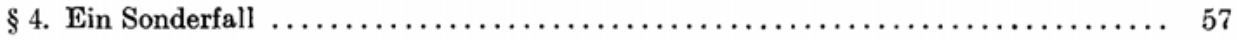

$\S 5$. Abschätzung der Anzahl linear separierbarer Dichotomien $\ldots \ldots \ldots \ldots \ldots \ldots \ldots \ldots \ldots$

$\S 6$. Bedingungen für die gleichmäßige Konvergenz der Ereignishäufigkeiten gegen ihre

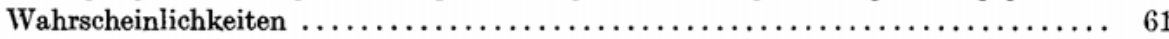

§ 7. Eigenschaften der Wachstumsfunktion $\ldots \ldots \ldots \ldots \ldots \ldots \ldots \ldots \ldots \ldots \ldots \ldots \ldots \ldots \ldots \ldots \ldots \ldots$

§ 8. Schätzung der Abweichung der empirisch-optimalen Entscheidungsregel von der

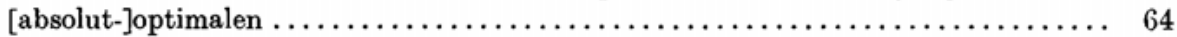

§ 9. Methode der Minimierung der empirischen Kosten im Fall der deterministischen Pro-

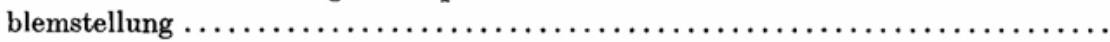

\$10. Bemerkungen zur Schätzung der Geschwindigkeit der gleichmäßigen Konvergenz von Ereignishäufigkeiten gegen ihre Wahrscheinlichkeiten $\ldots \ldots \ldots \ldots \ldots \ldots \ldots \ldots, 68$

$\S 11$. Bemerkungen zur Minimierung der empirischen Kosten $\ldots \ldots \ldots \ldots \ldots \ldots \ldots \ldots, 71$

$\S 12$. Algorithmen der Methode des generalisierten Porträts $\ldots \ldots \ldots \ldots \ldots \ldots \ldots \ldots \ldots, 72$

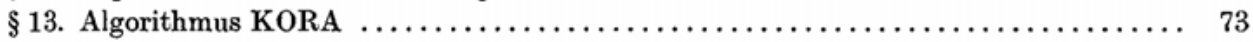

Kapitel VI. Die Methode der geordneten Kostenminimierung $\ldots \ldots \ldots \ldots \ldots \ldots \ldots \ldots \ldots \ldots$

$\S 1$ Die Kriterien der Güteschätzung für Algorithmen $\ldots \ldots \ldots \ldots \ldots \ldots \ldots \ldots \ldots \ldots, 76$

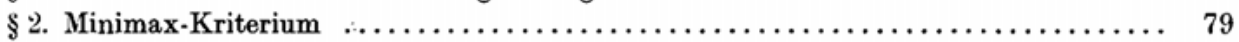

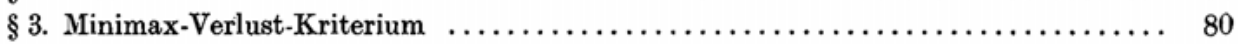

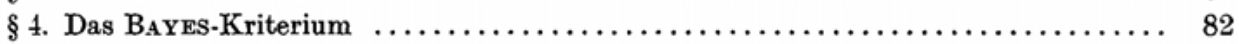

§ 5. Verein von Klassen von Entscheidungsregeln (halbgeordnetes Mengensystem) ..... 83

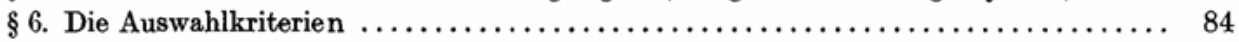

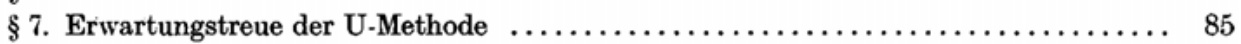

§. Rangordnung nach Merkmalen (Aufbauverfahren) $\ldots \ldots \ldots \ldots \ldots \ldots \ldots \ldots \ldots \ldots \ldots$

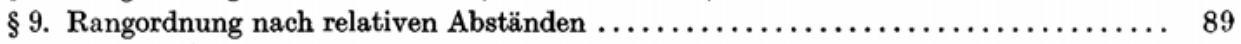

\$10. Rangordnung auf der Basis empirischer Schätzungen des relativen Abstandes und Problem der Minimierung der summarischen Kosten ..................... 93

$\S 11$. Uber die Auswahl eines optimalen Satzes von Merkmalen $\ldots \ldots \ldots \ldots \ldots \ldots \ldots \ldots .98$

\$12. Algorithmen der geordneten Suche des Minimums der summarischen Kosten ........ 101

§13. Konstruktionsalgorithmen für extremale stückweise lineare Entscheidungsregeln $\ldots 103$

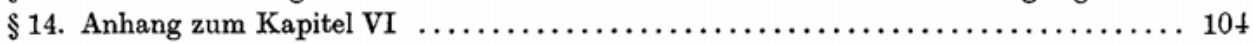


§ 1. Zur Unterscheidung erdölhaltiger und wasserhaltiger Schichten in der geologischen

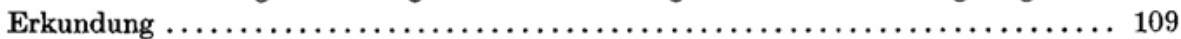

§ 2. Das Problem der Identifizierung von Handschriften $\ldots \ldots \ldots \ldots \ldots \ldots \ldots \ldots \ldots \ldots \ldots \ldots \ldots \ldots$

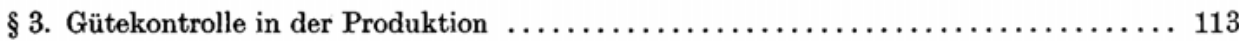

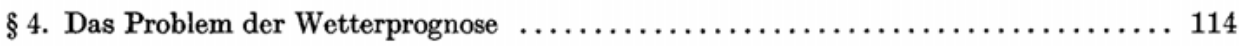

$\S 5$. Anwendung von Lernmethoden in der Medizin $\ldots \ldots \ldots \ldots \ldots \ldots \ldots \ldots \ldots \ldots \ldots \ldots \ldots \ldots$

$\S 6$. Einige Bemerkungen zum Einsatz von Lernmethoden $\ldots \ldots \ldots \ldots \ldots \ldots \ldots \ldots \ldots \ldots$

Kapitel VIII. Einige allgemeine Bemerkungen $\ldots \ldots \ldots \ldots \ldots \ldots \ldots \ldots \ldots \ldots \ldots \ldots \ldots \ldots 122$

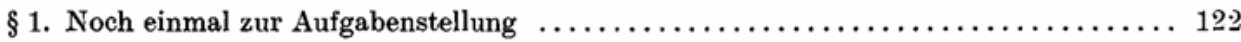

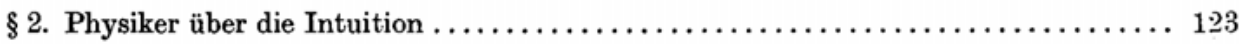

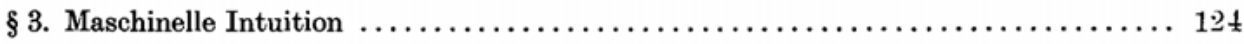

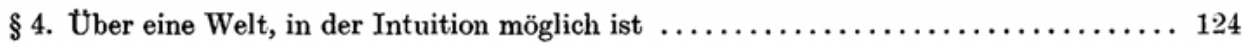

TEIL 2

\section{Statistische Grundlagen}

Kapitel IX. Die Konvergenz iterativer Algorithmen der Belehrung von Erkennungssystemen 128

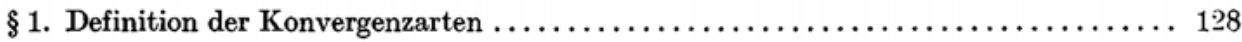

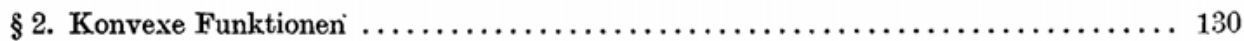

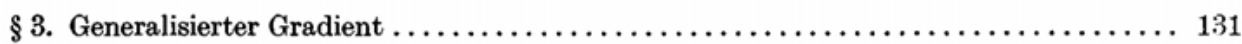

$\S 4$. Die Konvergenzbedingungen für iterative Algorithmen $\ldots \ldots \ldots \ldots \ldots \ldots \ldots \ldots \ldots \ldots$

$\S 5$. Eine weitere Bedingung für die Konvergenz von Iterationsalgorithmen $\ldots \ldots \ldots \ldots \ldots$

Kapitel X. Hinreichende Bedingungen für die gleichmäßige Konvergenz der Häufigkeiten gegen die Wahrscheinlichkeiten in einer Klasse von Ereignissen $\ldots \ldots \ldots \ldots \ldots \ldots \ldots \ldots \ldots \ldots$

§ 1. Die Abweichung des empirischen Kostenminimums vom erwarteten Kostenminimum 143

§ 2. Die Definition der gleichmäßigen Konvergenz der relativen Häufigkeiten gegen die

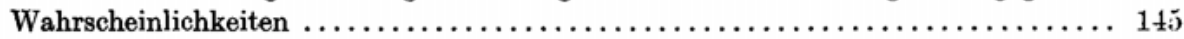

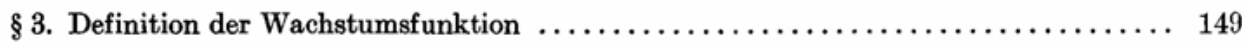

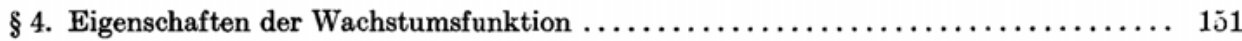

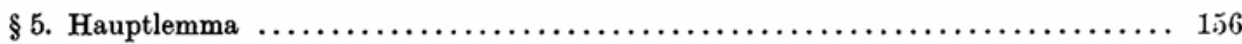

§ 6. Ableitung der hinreichenden Bedingungen für die gleichmäßige Konvergenz der Häufigkeiten gegen die Wahrscheinlichkeiten in einer Ereignisklasse $S \ldots \ldots \ldots \ldots \ldots \ldots$

§ 7. Uber die gleichmäßige Konvergenz mit Wahrscheinlichkeit Eins $\ldots \ldots \ldots \ldots \ldots \ldots 164$

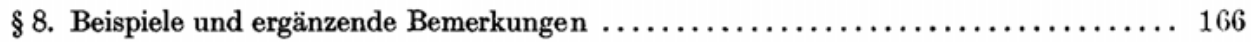

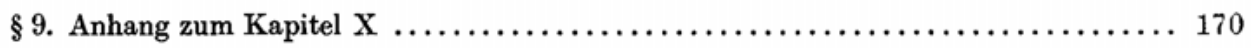


Kapitel XI. Notwendige und hinreichende Bedingungen für die gleichmäßige Konvergenz der Häufigkeiten gegen die Wahrscheinlichkeiten in einer Klasse von Ereignissen ..... 174

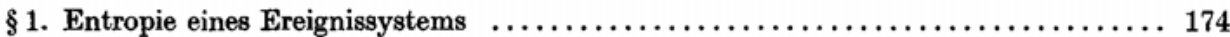

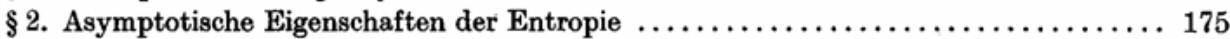

$\S 3$. Notwendige und hinreichende Bedingungen für die gleichmäßige Konvergenz (Beweis

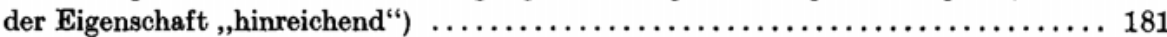

$\S 4$. Beweis der Notwendigkeit dieser Bedingungen für das Vorliegen der gleichmäßigen

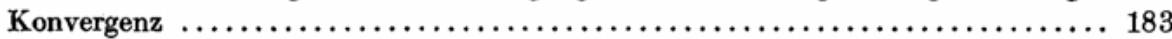

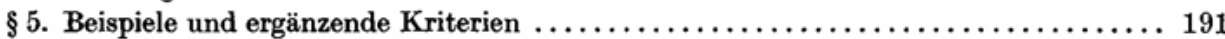

Kapitel XII. Schätzung der gleichmäßigen Abweichung der relativen Häufigkeiten von den Wahrscheinlichkeiten in einer Klasse von Ereignissen ................... 196

§1. Der Begriff gleichmäßige Abweichung ............................. 196

§ 2. Die Schätzung der gleichmäßigen Abweichung der relativen Häufigkeiten in zwei

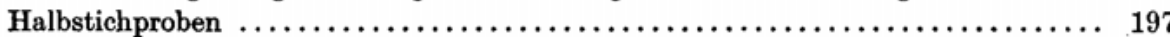

§ 3. Die Schätzung der gleichmäßigen Abweichung der relativen Häufigkeiten von den

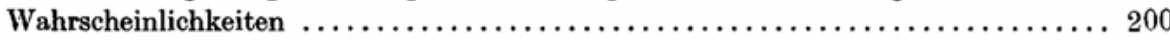

Kapitel XIII. Anwendung der Theorie der gleichmäßigen Konvergenz auf die Methoden der Kostenminimierùng $\ldots \ldots \ldots \ldots \ldots \ldots \ldots \ldots \ldots \ldots \ldots \ldots \ldots, 203$

§1. Abschätzung der hinreichenden Länge einer Lernfolge für die lernabhängige Er-

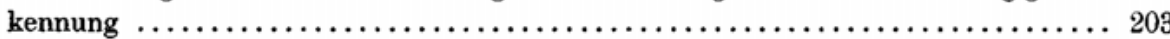

§ 2. Gleichmäßige Konvergenz der Mittelwerte gegen die mathematischen Erwartungen .. 210

TEIL 3

Methoden zur Konstruktion von Trennflächen

Kapitel XIV. Die Konstruktion der Trennhyperebene (Methode des generalisierten Porträts) .. 216

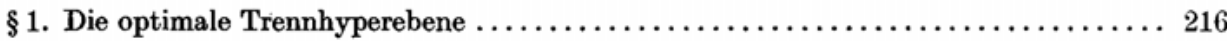

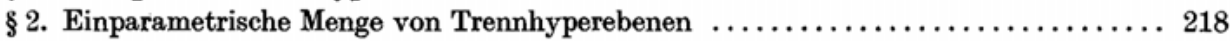

$\S 3$. Einige Eigenschaften des generalisierten Porträts ...................... 222

$\S 4$. Das generalisierte Porträt als Lösung eines Problems der quadratischen Programmierung $\ldots \ldots \ldots \ldots \ldots \ldots \ldots \ldots \ldots \ldots \ldots \ldots \ldots \ldots \ldots \ldots \ldots \ldots \ldots \ldots \ldots \ldots \ldots \ldots \ldots, 224$

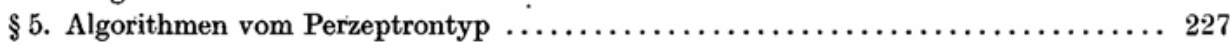

\$6. Gradientenmethoden zur Konstruktion der Trennhyperebene (Ermittlung des genera-

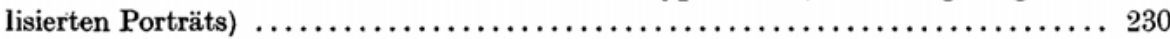

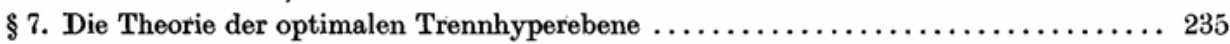

§ 8. Die optimale Trennhyperebene als Lösung eines Problems der quadratischen Program-

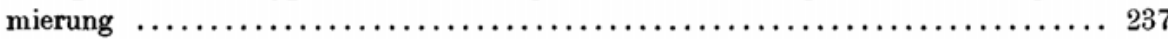

§ 9. Methoden zur numerischen Berechnung der optimalen Trennhy̆perebene $\ldots \ldots \ldots \ldots 239$

\$10. Konstruktion der optimalen Trennhyperebene nach einer modifizierten GAUSS-SEIDEL-

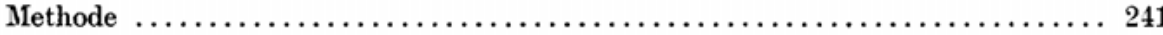


§11. Die Anwendung der Methode des generalisierten Porträts zur Ermittlung der optima-

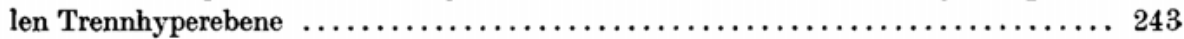

§12. Einige statistische Besonderheiten der Methode des generalisierten Porträts ....... 244

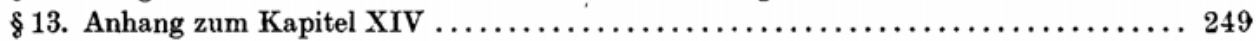

Kapitel XV. Lernabhängige Erkennungsalgorithmen zur Realisierung der Methode des genera-

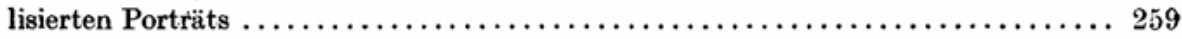

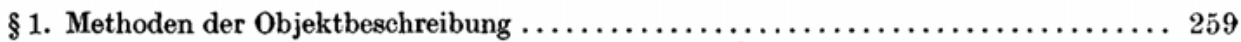

$\S 2$. Algorithmus zur Konstruktion der Trennhyperebene $\ldots \ldots \ldots \ldots \ldots \ldots \ldots \ldots \ldots \ldots 262$

§3. Konstruktionsalgorithmus für eine Trennhyperebene zur Minimierung der Anzahl

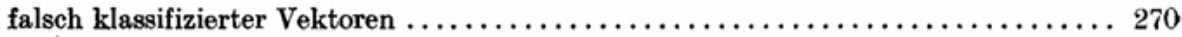

§4. Algorithmus zur Konstruktion einer stückweise linearen Trennfläche $\ldots \ldots \ldots \ldots \ldots 271$

§5. Algorithmen zur Konstruktion der Trennhyperebene im Raum minimaler Dimension 272

$\S 6$. Algorithmus zur Konstruktion der extremalen Trennhyperebene . . . . . . . . . 274

§ 7. Algorithmus zur Konstruktion einer extremalen, stückweise linearen Trennhyper-

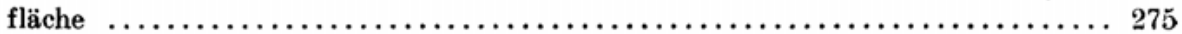

§ 8. Algorithmus zur Konstruktion einer Trennhyperebene unter Güteschätzung nach der

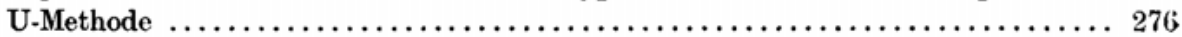

§9. Algorithmen zur Konstruktion einer extremalen Trennhyperebene mit Hilfe der

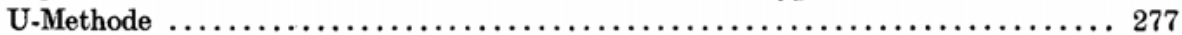

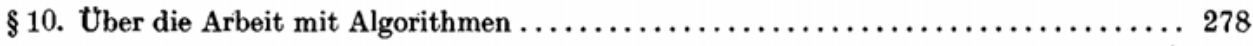

Kapitel XVI. Die Methode der konjugierten Richtungen $\ldots \ldots \ldots \ldots \ldots \ldots \ldots \ldots \ldots \ldots \ldots, 280$

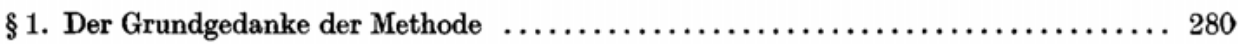

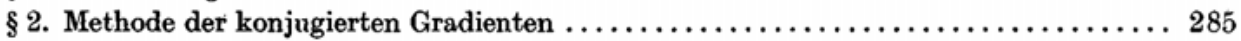

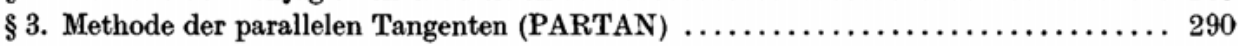

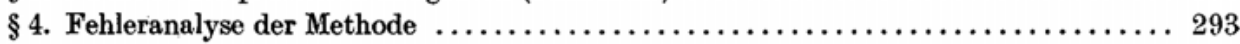

Kapitel XVII. Eine Lernmethode zur Ermittlung der Klassenentscheidung für eine Menge vorgegebener Objektvektoren (Neue Ergebnisse der Autoren als Ergänzung zur deutschen

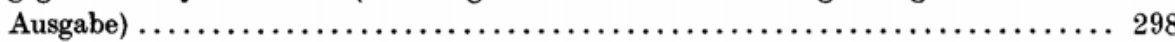

§1. Das Problem der Bestimmung der Funktionswerte in gegebenen Punkten ....... 298

§ 2. Minimierungsverfahren für die mittleren und für die summarischen Kosten ....... 299

$\S 3$. Schätzungen für die gleichmäßige Abweichung der Häufigkeiten in zwei Halbstich-

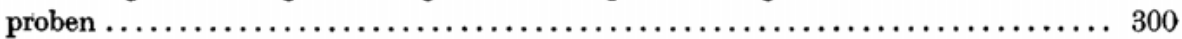

$\S 4$. Geordnete Minimierung der summarischen Kosten in einer Klasse linearer Entschei-

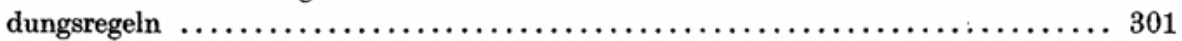

§ 5. Aussonderung von Objektvektoren aus der Stichprobe $\ldots \ldots \ldots \ldots \ldots \ldots \ldots \ldots \ldots \ldots$

$\S 6$. Geordnete Suche des Minimums der summarischen Kosten in der Klasse stückweise -

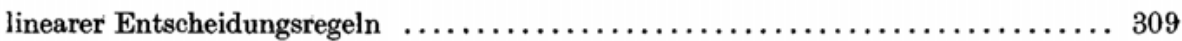

§ 7. ,Lokale Algorithmen " zur Minimierung der summarischen Kosten ............ 311

§8. Anwendung der Methode der geordneten Minimierung der summarischen Kosten ... 313

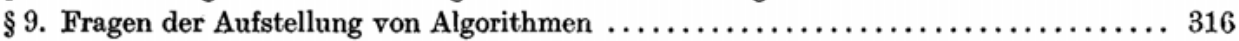




\section{Inhaltsverzeichnis}

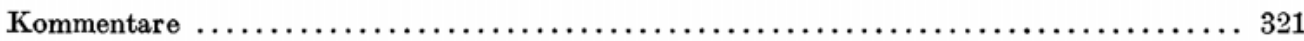

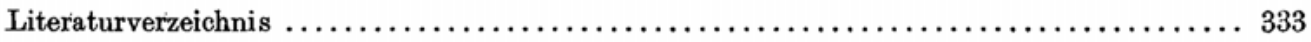

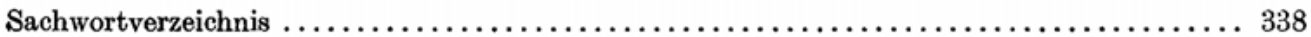

